\title{
Intérêt des gants radio-atténuateurs en radiologie interventionnelle : une évaluation expérimentale
}

\author{
J. GUERSEN ${ }^{1}$, L. DONADILLE ${ }^{2}$, J.L. REHEL ${ }^{2}$, A. CHARVAIS ${ }^{1}$, \\ R. ZAKNOUNE ${ }^{2}$, L. CASSAGNES ${ }^{1}$, P. CHABROT ${ }^{1}$, L. BOYER ${ }^{1}$
}

(Manuscrit reçu le 7 avril 2011, accepté le 26 avril 2011)

RÉSUMÉ Objectif : déterminer l'intérêt des gants radio-atténuateurs plombés pour diminuer l'exposition des mains des opérateurs, en radiologie interventionnelle et au bloc opératoire. Matériel et méthode : on a utilisé des gants radio-atténuateurs plombés du marché, des dosimètres TLD thermoluminescents, un fantôme anthropomorphe de calibration en ostéodensitométrie, une main anthropomorphe. Les mesures étaient réalisées sur 2 installations : l'une avec capteurs plans, l'autre avec amplificateur de brillance. On a comparé les doses reçues par dosimètres sans et dans un gant, dans le champ direct (10 séries de mesures dont 2 avec amplificateur de brillance) et dans le diffusé ( 3 séries sur capteurs plans), en scopie et graphie. Résultats : dans le diffusé, les dosimètres dans le gant mesuraient une diminution de dose, variant de 1 à 3 . Dans le champ direct, 7 mesures sur 10 montraient une augmentation de dose dans le gant (13 à $42 \%)$. 3/10 mesures montraient une quasi stabilité de dose avec le gant $(-3,3 \%$ à $+2 \%)$. Conclusion : nos mesures objectivent une efficacité des gants radio-atténuateurs plombés dans le diffusé. Dans le rayonnement direct, le port des gants radio-atténuateurs plombés entraîne une augmentation de la dose - main, du fait des réglages automatiques des paramètres.

ABSTRACT Potential of radiation attenuation gloves in interventional radiology: an experimental evaluation.

Objective: to determine the potential of leaded radiation attenuation gloves to decrease the X-ray exposure of operators' hands, in interventional radiology and in surgical units. Material and method: we used: radiation attenuation gloves bought by our hospital, TLD thermoluminescent dosimeters, an anthropomorphic phantom of calibration in bone densitometry, an anthropomorphic hand. The measurements were realized on 2 installations: one with sensor plans, and the other one with a brightness amplifier. We compared doses received by dosimeters without and in a glove, in the direct field (10 series of measurements, 2 with the brightness amplifier) and in the diffuse field ( 3 series on sensor plans), in fluoroscopy and graphy. Results: in the diffuse field, dosimeters in the glove measured a decrease in dose, varying from 1 to 3 . In the direct field, 7 measurements out of 10 showed an increase in dose in the glove (13 to $42 \%)$. 3/10 measurements showed an almost stable dose. Conclusion: our measurements show the efficiency ofleaded radiation attenuation gloves in the diffuse field. In the direct field, the radiation attenuation gloves increase the hand dose, because of the automatic tuning of the parameters.

Keywords: Radio-attenuators gloves / interventional radiology / efficiency / interest

1 CHU Clermont Ferrand Pôle Imagerie Médicale, 58 rue Montalembert, 63003 Clermont-Ferrand Cedex 1, France.

2 IRSN, DRPH, BP 17, 92262 Fontenay-aux-Roses, France. 


\section{Introduction}

Depuis plusieurs années, le nombre d'examens radiologiques interventionnels et de procédures opératoires guidées par rayons $\mathrm{X}$ est en constante augmentation. L'intérêt de ces examens est aujourd'hui indiscutable. Mais, outre le risque déterministe et stochastique, non négligeable pour les patients, lié aux doses significatives de rayons $\mathrm{X}$ qu'ils peuvent recevoir, la mise en œuvre de ces procédures requiert, pour les opérateurs directs, tout un arsenal individuel et collectif de protections destinées à limiter le plus possible l'exposition au rayonnement diffusé émis pendant la réalisation des examens. Les mains des opérateurs, qui reçoivent les doses de rayonnement diffusé les plus élevées, auxquelles s'ajoute le rayonnement direct lorsqu'elles « passent » dans le faisceau principal, n'échappent pas à cette règle. L'utilisation de gants radio-atténuateurs plombés, souples et à usage unique (stériles) apparaît naturellement, jusqu'à présent, comme une solution à la problématique de l'exposition des mains aux rayons $\mathrm{X}$.

Il existe toutefois un doute sur l'efficacité réelle de ces types de produits, en particulier dans le champ primaire d'exposition, avec l'asservissement des paramètres de production d'image à la densité des structures traversées. L'atténuation du rayonnement par les gants atténuateurs plombés (principal argument de leur utilisation) n'est-elle pas effacée, voire dépassée, par l'augmentation des paramètres - machine, liés à la présence dans le champ d'exposition d'une structure plus dense ?

Devant cette légitime interrogation sur le bien fondé de l'utilisation de ces protections coûteuses, nous avons souhaité évaluer l'apport réel des gants radioatténuateurs plombés utilisés par les radiologues en radiologie interventionnelle, en comparant les doses de rayons $\mathrm{X}$ reçues par des dosimètres positionnés à l'intérieur d'un gant et utilisés sans gant, avec un fantôme anthropomorphe, dans différentes situations, et dans deux salles différentes de radiologie interventionnelle.

\section{Matériel et méthode}

\subsection{Matériel utilisé}

Pour cette étude, nous avons utilisé les gants radio-atténuateurs plombés disponibles au CHU de Clermont-Ferrand, et correspondant au marché en cours : gants antiradiations (radiation reducing glove) de marque PROTECH PROGUARD RR ${ }^{\mathrm{TM}}$ - Model RR-2. 
Les dosimètres utilisés pour réaliser les mesures étaient des dosimètres TLD thermo luminescents.

Pour simuler le patient et générer du rayonnement diffusé pendant les mesures, nous avons positionné, sur les tables d'examen des salles de radiologie interventionnelles, un fantôme anthropomorphe utilisé habituellement pour la calibration d'un ostéo densitomètre (Hologic - X Ray Bone Densitometer QDR 4500 -1996).

Caractéristiques du fantôme : longueur $=17,8 \mathrm{~cm}$; largeur $=15,3 \mathrm{~cm}$; hauteur $=17,3 \mathrm{~cm} ;$ surface $=52,78 \mathrm{~cm}^{2} ;$ densité $=0,998$ ).

La dernière série de mesures a été réalisée avec une main anthropomorphe mise à disposition par l'IRSN.

Les mesures se sont déroulées sur une installation de marque Philips (Integris Allura FD20 monoplan - 2006) équipée de capteurs plans, et une installation de marque Siemens (Multistar Top Polytron monoplan - 1999) équipée d'un amplificateur de brillance.

\subsection{Méthode (voir Figs. 1, 2, 3 et 4)}

Pour objectiver l'efficacité des gants radio-atténuateurs utilisés, nous avons procédé à des mesures comparatives des doses de rayons $\mathrm{X}$ reçues par 2 dosimètres TLD :

(1) positionnés directement sur le fantôme, sans gant, dans le champ primaire d'exposition (tube sous la table d'examen) en mode scopie et en mode graphie,

(2) positionnés dans un gant, posé sur le fantôme, dans le champ primaire, d'exposition (tube sous la table d'examen) en mode scopie et en mode graphie,

(3) positionnés sur la table d'examen, sans gant, à $15 \mathrm{~cm}$ du bord du fantôme dans le rayonnement diffusé (tube sous la table d'examen) en mode scopie et en mode graphie,

(4) positionnés dans un gant, à $15 \mathrm{~cm}$ du bord du fantôme, dans le rayonnement diffusé (tube sous la table d'examen) en mode scopie et en mode graphie.

Les situations (1) et (2) (champ primaire d'exposition) ont donné lieu, avec l'installation Philips capteurs plans, à 3 séries de mesures, et à 1 série de mesures avec l'installation Siemens équipée d'un amplificateur de brillance.

Les conditions (3) et (4) (rayonnement diffusé) ont donné lieu, sur l'installation Philips capteurs plans, à 1 série de mesures en scopie et en graphie. Ces conditions n'ont pas été testées sur l'installation équipée d'un amplificateur de brillance. 
J. GUERSEN et al.

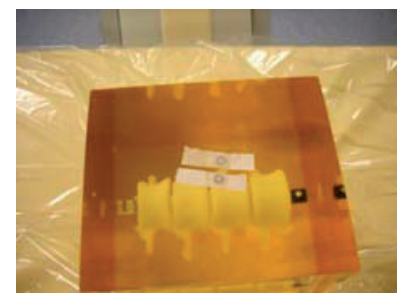

Figure 1 - Dosimètres positionnés directement sur le fantôme, sans gant, dans le champ primaire d'exposition (tube sous la table d'examen).

Dosimeters positioned directly on the phantom, without glove, in the primary field of exposure (tube under the table).

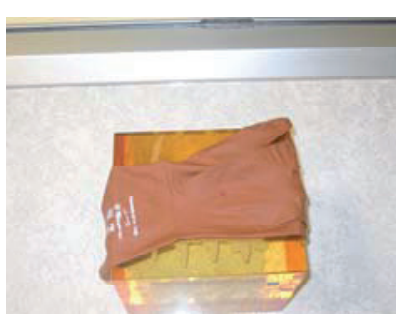

Figure 2 -Dosimètres positionnés dans un gant, posé sur le fantôme, dans le champ primaire d'exposition (tube sous la table d'examen).

Dosimeters positioned in a glove, landed on the Phantom, in the primary field of exposure (tube under the table).

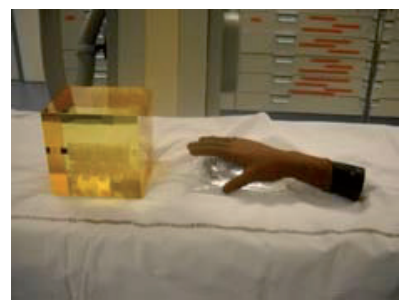

Figure 3 -Dosimètres positionnés sur la table d'examen dans un gant, sur une main anthropomorphe, à $15 \mathrm{~cm} \mathrm{du}$ bord du fantôme dans le rayonnement diffusé (tube sous la table d'examen).

Dosimeters positioned on the table in a glove on an anthropomorphic hand, $15 \mathrm{~cm}$ from the edge of the hand in the scattered radiation (tube under the table).

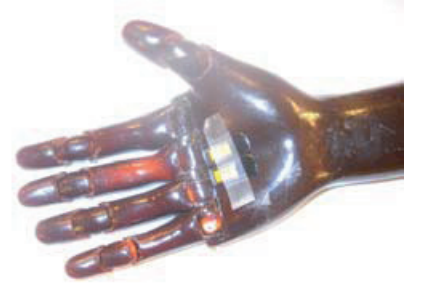

Figure 4-Dosimètres positionnés sur la main anthropomorphe.

Dosimeters positioned on the anthropomorphic hand.

Conditions (5) et (6) : une série de mesures complémentaire a été réalisée avec une main anthropomorphe, sur l'installation Philips équipée de capteurs plans, en scopie et en graphie, dans le faisceau et dans le rayonnement diffusé. Ces conditions n'ont pas été testées sur l'installation Siemens équipée d'un amplificateur de brillance. 
Les différentes séries de mesures ont été opérées avec des paramètres fixes identiques, permettant des comparaisons (DFI, DFP, champ, collimation, durée). Dans tous les cas, le réglage des paramètres de production d'image (kilo-voltage et milli-ampérage) était en mode automatique.

À noter qu'entre la seconde et la troisième série, l'installation a bénéficié d'une intervention technique d'optimisation dosimétrique.

\section{Résultats}

Ils figurent sur les tableaux I, II et III.

\subsection{Dans le rayonnement diffusé, à paramètres constants}

\subsubsection{En mode scopie}

La série de mesures réalisée dans la salle Philips capteurs plans, a montré l'absence de variation de la dose mesurée par le dosimètre TLD, qu'il soit posé directement sur la table d'examen à $15 \mathrm{~cm}$ du bord du fantôme, ou qu'il soit à l'intérieur d'un gant radio-atténuateur (au même endroit sur la table). Cette mesure n'a donc pas démontré l'efficacité des gants, mais elle n'a été réalisée qu'une seule fois.

La série de mesures réalisée avec une main anthropomorphe, a montré, pour une tension donnée $(77 \mathrm{kV})$, une réduction de dose d'un facteur 3 avec les gants radio-atténuateurs.

\subsubsection{En mode graphie}

La mesure réalisée a objectivé une diminution de $12 \%$ de la dose enregistrée par le dosimètre protégé dans le gant. Cette mesure n'a été réalisée qu'une seule fois L'efficacité du gant radio-atténuateur est apparue comme marginale.

\subsection{Dans le champ primaire d'exposition aux rayons $X$}

\subsubsection{En mode scopie}

$\rightarrow 4$ séries de mesures ont été réalisées dans la salle Philips capteurs plans :

- La première, avec 1 dosimètre, a affiché une augmentation de $14 \%$ de la dose mesurée lorsque le dosimètre était placé dans un gant. Cette augmentation suivait l'augmentation en parallèle du kilovoltage affiché $(80 \mathrm{kV}$ versus $78 \mathrm{kV})$. 
TABLEAU I

Mesures réalisées sur l'installation Philips équipée de capteurs plans - 2006. Measurements with sensor plans Philips equipment - 2006.

\begin{tabular}{|c|c|c|c|c|c|c|}
\hline & \multicolumn{2}{|c|}{$\begin{array}{l}\text { Mesures dans le champ primaire } \\
\text { d'exposition }\end{array}$} & \multirow[t]{2}{*}{ Variation } & \multicolumn{2}{|c|}{$\begin{array}{l}\text { Mesures réalisées dans } \\
\text { le rayonnement diffusé }\end{array}$} & \multirow[t]{2}{*}{ Variation } \\
\hline & $\begin{array}{c}\text { Dosimètres } \\
\text { sans gant }\end{array}$ & $\begin{array}{l}\text { Dosimètres } \\
\text { dans le gant }\end{array}$ & & $\begin{array}{c}\text { Dosimètres } \\
\text { sans gant }\end{array}$ & $\begin{array}{l}\text { Dosimètres } \\
\text { dans le gant }\end{array}$ & \\
\hline $\begin{array}{l}1^{\mathrm{re}} \text { série } \\
\text { de mesures } \\
\text { janvier } 2010\end{array}$ & $\begin{array}{c}\text { Dose } \\
/ 1 \text { dosimètre }\end{array}$ & $\begin{array}{c}\text { Dose } \\
\text { /1 dosimètre }\end{array}$ & & $\begin{array}{c}\text { Dose } \\
\text { /1 dosimètre }\end{array}$ & $\begin{array}{c}\text { Dose } \\
\text { /1 dosimètre }\end{array}$ & \\
\hline Mode scopie & $0,48 \mathrm{mSv}$ & $0,55 \mathrm{mSv}$ & $\uparrow 14 \%$ & $0,35 \mathrm{mSv}$ & $0,35 \mathrm{mSv}$ & $\begin{array}{l}\text { pas de } \\
\text { variation }\end{array}$ \\
\hline $\begin{array}{l}\text { Paramètres } \\
\text { d'acquisition }\end{array}$ & $\begin{array}{l}2 \text { minutes; } \\
78 \mathrm{kV} 8,7 \mathrm{~mA}\end{array}$ & $\begin{array}{l}2 \text { minutes; } \\
80 \mathrm{kV} 8,7 \mathrm{~mA}\end{array}$ & & $\begin{array}{l}2 \text { minutes; } \\
78 \mathrm{kV} 8,7 \mathrm{~mA}\end{array}$ & $\begin{array}{l}2 \text { minutes; } \\
78 \mathrm{kV} 8,6 \mathrm{~mA}\end{array}$ & \\
\hline Mode graphie & $0,62 \mathrm{mSv}$ & $0,77 \mathrm{mSv}$ & $\uparrow 24 \%$ & $1,55 \mathrm{mSv}$ & $1,34 \mathrm{mSv}$ & $\uparrow 12 \%$ \\
\hline $\begin{array}{l}\text { Paramètres } \\
\text { d'acquisition }\end{array}$ & $\begin{array}{l}10 \text { secondes; } \\
80 \mathrm{kV} 9 \mathrm{mAs}\end{array}$ & $\begin{array}{l}10 \text { secondes; } \\
80 \mathrm{kV} 12 \mathrm{mAs}\end{array}$ & & $\begin{array}{l}30 \text { secondes; } \\
80 \mathrm{kV} \quad 9 \mathrm{mAs}\end{array}$ & $\begin{array}{l}30 \text { secondes; } \\
80 \mathrm{kV} 9 \mathrm{mAs}\end{array}$ & \\
\hline $\begin{array}{l}2^{\mathrm{e}} \text { série } \\
\text { de mesures } \\
\text { mars } 2010\end{array}$ & $\begin{array}{l}\text { Dosemoyenne } \\
/ 2 \text { dosimètres }\end{array}$ & $\begin{array}{l}\text { Dosemoyenne } \\
12 \text { dosimètres }\end{array}$ & & $\begin{array}{l}\text { Dose moyenne } \\
12 \text { dosimètres }\end{array}$ & $\begin{array}{l}\text { Dose moyenne } \\
12 \text { dosimètres }\end{array}$ & \\
\hline Mode scopie & $1,0005 \mathrm{mSv}$ & $0,984 \mathrm{mSv}$ & $\uparrow 1,6 \%$ & non réalisé & non réalisé & \\
\hline $\begin{array}{l}\text { Paramètres } \\
\text { d'acquisition }\end{array}$ & $\begin{array}{l}4 \text { minutes; } \\
78 \mathrm{kV} 8,5 \mathrm{~mA}\end{array}$ & $\begin{array}{l}4 \text { minutes; } \\
80 \mathrm{kV} 8,9 \mathrm{~mA}\end{array}$ & & & & \\
\hline Mode graphie & $1,8495 \mathrm{mSv}$ & $1,891 \mathrm{mSv}$ & $\uparrow 2 \%$ & non réalisé & non réalisé & \\
\hline $\begin{array}{l}\text { Paramètres } \\
\text { d'acquisition }\end{array}$ & $\begin{array}{l}\text { 20secondes; } \\
80 \mathrm{kV} 8 \mathrm{mAs}\end{array}$ & $\begin{array}{l}\text { 20secondes; } \\
80 \mathrm{kV} 12 \mathrm{mAs}\end{array}$ & & & & \\
\hline $\begin{array}{l}3^{\mathrm{e}} \text { série de } \\
\text { mesures mai } 2010\end{array}$ & $\begin{array}{l}\text { Dosemoyenne } \\
/ 2 \text { dosimètres }\end{array}$ & $\begin{array}{l}\text { Dosemoyenne } \\
/ 2 \text { dosimètres }\end{array}$ & & $\begin{array}{l}\text { Dosemoyenne } \\
12 \text { dosimètres }\end{array}$ & $\begin{array}{l}\text { Dose moyenne } \\
/ 2 \text { dosimètres }\end{array}$ & \\
\hline Mode scopie & $0,723 \mathrm{mSv}$ & $0,930 \mathrm{mSv}$ & $\uparrow 29 \%$ & non réalisé & non réalisé & \\
\hline $\begin{array}{l}\text { Paramètres } \\
\text { d'acquisition }\end{array}$ & $\begin{array}{c}4 \text { minutes; } \\
80 \mathrm{kV} \quad 8,5 \mathrm{~mA}\end{array}$ & $\begin{array}{c}4 \text { minutes; } \\
80 \mathrm{kV} \quad 8,7 \mathrm{~mA}\end{array}$ & & & & \\
\hline Mode graphie & $1,6705 \mathrm{mSv}$ & $1,9915 \mathrm{mSv}$ & $\uparrow 19 \%$ & non réalisé & non réalisé & \\
\hline $\begin{array}{l}\text { Paramètres } \\
\text { d'acquisition }\end{array}$ & $\begin{array}{l}\text { 40 secondes; } \\
85 \mathrm{kV} 8 \mathrm{mAs}\end{array}$ & $\begin{array}{l}\text { 40secondes; } \\
85 \mathrm{kV} 11 \mathrm{mAs}\end{array}$ & & & & \\
\hline
\end{tabular}

- La seconde série (2 dosimètres) a fait apparaître une quasi stabilité de la dose (diminution non significative de 1,6\%), malgré une augmentation du kilovoltage et des milli ampères $(80 \mathrm{kV}$ versus $78 \mathrm{kV}$ et $8,9 \mathrm{~mA}$ versus $8,5 \mathrm{~mA}$ ).

- La troisième série (2 dosimètres) a objectivé une augmentation de la dose de $29 \%$, lorsque le dosimètre était positionné dans le gant. Le milli ampérage a augmenté également $(8,7 \mathrm{~mA}$ versus $8,5 \mathrm{~mA})$. 


\section{TABLEAU II}

Mesures sur l'installation Siemens équipée d'un amplificateur de brillance - 1999. Measurements with brightness amplifier Siemens equipment - 1999.

\begin{tabular}{|c|c|c|c|c|c|c|}
\hline & \multicolumn{2}{|c|}{$\begin{array}{l}\text { Mesures dans le champ primaire } \\
\text { d'exposition }\end{array}$} & \multirow[t]{2}{*}{ Variation } & \multicolumn{2}{|c|}{$\begin{array}{l}\text { Mesures réalisées dans } \\
\text { le rayonnement diffusé }\end{array}$} & \multirow[t]{2}{*}{ Variation } \\
\hline & $\begin{array}{c}\text { Dosimètres } \\
\text { sans gant }\end{array}$ & $\begin{array}{l}\text { Dosimètres } \\
\text { dans le gant }\end{array}$ & & $\begin{array}{l}\text { Dosimètres } \\
\text { sans gant }\end{array}$ & $\begin{array}{l}\text { Dosimètres } \\
\text { dans le gant }\end{array}$ & \\
\hline $\begin{array}{l}3^{\mathrm{e}} \text { série de mesures } \\
\text { mai } 2010\end{array}$ & $\begin{array}{l}\text { Dose moyenne } \\
/ 2 \text { dosimètres }\end{array}$ & $\begin{array}{l}\text { Dose moyenne } \\
12 \text { dosimètres }\end{array}$ & & $\begin{array}{l}\text { Dose moyenne } \\
12 \text { dosimètres }\end{array}$ & $\begin{array}{l}\text { Dose moyenne } \\
12 \text { dosimètres }\end{array}$ & \\
\hline Mode scopie & $1,017 \mathrm{mSv}$ & $1,441 \mathrm{mSv}$ & $\uparrow 42 \%$ & non réalisé & non réalisé & \\
\hline $\begin{array}{l}\text { Paramètres } \\
\text { d'acquisition }\end{array}$ & $\begin{array}{c}4 \text { minutes } \\
80 \mathrm{kV} 47 \mathrm{~mA} \\
3,6 \mathrm{~ms} ; 30 \mathrm{p} / \mathrm{s}\end{array}$ & $\begin{array}{c}4 \text { minutes } \\
80 \mathrm{kV} 56 \mathrm{~mA} \\
4,4 \mathrm{~ms} ; 30 \mathrm{p} / \mathrm{s}\end{array}$ & & & & \\
\hline Mode graphie & $3,739 \mathrm{mSv}$ & $4,215 \mathrm{mSv}$ & $\uparrow 13 \%$ & non réalisé & non réalisé & \\
\hline $\begin{array}{l}\text { Paramètres } \\
\text { d'acquisition }\end{array}$ & $\begin{array}{c}40 \text { secondes } \\
79 \mathrm{kV} ; 221 \mathrm{~mA} \\
122 \mathrm{~ms} ; 3 \mathrm{i} / \mathrm{s}\end{array}$ & $\begin{array}{c}40 \text { secondes; } \\
87 \mathrm{kV} ; 198 \mathrm{~mA} \\
122 \mathrm{~ms} ; 3 \mathrm{i} / \mathrm{s}\end{array}$ & & & & \\
\hline
\end{tabular}

TABLEAU III

Mesures réalisées avec une main anthropomorphe sur l'installation Philips équipée de capteurs plans - 2006.

Measurements with anthropomorphic hand Philips equipment with sensor plans - 2006.

\begin{tabular}{|c|c|c|c|c|c|c|}
\hline & \multicolumn{2}{|c|}{$\begin{array}{l}\text { Mesures dans le champ primaire } \\
\text { d'exposition }\end{array}$} & \multirow[t]{2}{*}{ Variation } & \multicolumn{2}{|c|}{$\begin{array}{l}\text { Mesures réalisées dans } \\
\text { le rayonnement diffusé }\end{array}$} & \multirow[t]{2}{*}{ Variation } \\
\hline & $\begin{array}{c}\text { Dosimètres } \\
\text { sans gant }\end{array}$ & $\begin{array}{l}\text { Dosimètres } \\
\text { dans le gant }\end{array}$ & & $\begin{array}{l}\text { Dosimètres } \\
\text { sans gant }\end{array}$ & $\begin{array}{l}\text { Dosimètres } \\
\text { dans le gant }\end{array}$ & \\
\hline $\begin{array}{l}4^{\mathrm{e}} \text { série de mesures } \\
\text { janvier } 2011\end{array}$ & $\begin{array}{c}\text { Dose } \\
/ 2 \text { dosimètres }\end{array}$ & $\begin{array}{c}\text { Dose } \\
/ 2 \text { dosimètres }\end{array}$ & & $\begin{array}{c}\text { Dose } \\
/ 2 \text { dosimètres }\end{array}$ & $\begin{array}{c}\text { Dose } \\
/ 2 \text { dosimètres }\end{array}$ & \\
\hline Mode scopie & $1,40 \mathrm{mSv}$ & $1,66 \mathrm{mSv}$ & $\uparrow 18 \%$ & $0,31 \mathrm{mSv}$ & $0,10 \mathrm{mSv}$ & $\begin{array}{l}\text { Diminution } \\
\text { facteur } 3\end{array}$ \\
\hline $\begin{array}{l}\text { Paramètres } \\
\text { d'acquisition }\end{array}$ & $\begin{array}{l}3 \text { minutes } \\
83 \mathrm{kV} 8,7 \mathrm{~mA}\end{array}$ & $\begin{array}{l}3 \text { minutes ; } \\
86 \mathrm{kV} \quad 8,5 \mathrm{~mA}\end{array}$ & & $\begin{array}{c}4 \text { minutes ; } \\
77 \mathrm{kV} ; 8,3 \mathrm{Ma}\end{array}$ & $\begin{array}{c}4 \text { minutes } \\
77 \mathrm{kV} ; 8,3 \mathrm{Ma}\end{array}$ & \\
\hline Mode graphie & $3,02 \mathrm{mSv}$ & $2,92 \mathrm{mSv}$ & $\uparrow 3,3 \%$ & non réalisé & non réalisé & \\
\hline $\begin{array}{l}\text { Paramètres } \\
\text { d'acquisition }\end{array}$ & $\begin{array}{l}\text { 40 secondes; } \\
85 \mathrm{kV} \quad 17 \mathrm{mAs}\end{array}$ & $\begin{array}{l}40 \text { secondes; } \\
85 \mathrm{kV} \quad 21 \mathrm{mAs}\end{array}$ & & & & \\
\hline
\end{tabular}

- La quatrième série de mesures a objectivé une augmentation de la dose de $18 \%$, lorsque le dosimètre était positionné dans le gant. Le kilovoltage a augmenté également ( $86 \mathrm{kV}$ versus $83 \mathrm{kV})$ et le milliampérage a diminué (8,5 $\mathrm{mA}$ versus $8,7 \mathrm{~mA})$.

$\rightarrow 1$ série de mesures ( 2 dosimètres) a été réalisée dans la salle Siemens avec amplificateur brillance :

- Elle a fait apparaître une augmentation de $42 \%$ de la dose mesurée dans le cas du dosimètre positionné dans le gant radio-atténuateur, associée à une augmentation des paramètres (56 mA versus $47 \mathrm{~mA}$ et 4,4 ms versus 3,6 ms). 


\subsubsection{En mode graphie}

$\rightarrow 4$ séries de mesures ont été réalisées dans la salle Philips capteurs plans :

- La première série a affiché une augmentation de $24 \%$ de la dose, lorsque le dosimètre était dans le gant, associée à une augmentation des paramètres (12 $\mathrm{mAs}$ versus $9 \mathrm{mAs}$ ).

- La seconde a fait apparaître une quasi stabilité de la dose (diminution non significative de $2 \%)$, malgré une augmentation des mAs (12 mAs versus $8 \mathrm{mAs}$ ).

- La troisième a objectivé une augmentation de $19 \%$, parallèlement à l'augmentation des mAs (11 mAs versus $8 \mathrm{mAs}$ ).

- La quatrième série a fait apparaître une quasi stabilité de la dose (diminution non significative de 3,3\%), malgré une augmentation des mAs (21 mAs versus $17 \mathrm{mAs})$.

$\rightarrow 1$ série de mesures ( 2 dosimètres) a été réalisée dans la salle Siemens avec amplificateur brillance :

- Elle a montré une augmentation de $13 \%$; les paramètres étaient également modifiés : le kilovoltage augmentait $(87 \mathrm{kV}$ versus $79 \mathrm{kV})$; les milliampères diminuaient (198 mA versus $221 \mathrm{~mA}$ ).

Au total, dans le champ primaire d'exposition aux rayons $\mathrm{X}$, on a assisté, en mode scopie, à une augmentation de la dose mesurée lorsque le dosimètre était placé dans un gant radio-atténuateur plombé ; cette augmentation variait de 14 à $42 \%$ selon les tests et l'installation choisie. Elle résultait de l'augmentation des paramètres. Les séries qui indiquaient une diminution de 1,6 \% ne semblaient pas devoir être retenues, puisque les $\mathrm{kV}$ et les mA affichés augmentaient lorsqu'il y avait utilisation des gants, et que la dose mesurée augmentait dans 2 autres séries, même lorsqu'un seul des paramètres augmentait. De plus, l'incertitude des résultats avec les dosimètres était de l'ordre de $10 \%$.

- En graphie, l'utilisation d'un gant radio-atténuateur plombé générait une augmentation de la dose, qui variait entre 13 et $24 \%$ selon les tests et l'installation choisie. Cette augmentation résultait de l'augmentation des paramètres. Les séries qui indiquaient une quasi stagnation de la dose (augmentation de $2 \%$ ou diminution de 3,3\%) ne devaient pas être retenues, pour les raisons évoquées précédemment.

- Dans le rayonnement diffusé, les mesures ont fait apparaître au mieux une diminution de la dose d'un facteur 3 avec les dosimètres placés dans un gant, et au pire une dose identique. 


\section{Discussion}

\subsection{Le contexte}

Au même titre que les autres protections plombées individuelles ou collectives, les gants radio atténuateurs sont utilisés par les praticiens, en radiologie interventionnelle et au bloc opératoire, pour diminuer l'exposition des mains aux rayons X. Au CHU de Clermont Ferrand, la consommation de ce type de protections a explosé ces 3 dernières années (155 paires en 2007, 280 paires en 2008, 800 paires en 2009), et le coût de ce poste de dépense, dans un environnement économique très contraint, est significatif (26 k€ en 2009).

Les procédures au cours desquelles les mains « passent » inévitablement dans le champ direct d'exposition (telles que les fistulographies artério-veineuses), qui sont un critère d'utilisation des gants radio-atténuateurs, nous ont interpellé sur le niveau réel d'efficacité de ces gants avec le réglage automatique des paramètres d'exposition.

\subsection{Nos résultats}

Notre étude expérimentale montre que, dans le rayonnement diffusé, les gants radio atténuateurs permettent une diminution de la dose, et que leur utilisation dans le champ direct d'exposition, induit une augmentation de la dose.

L'interprétation de nos résultats doit néanmoins tenir compte du fait que :

- les mesures avec les dosimètres TLD ont une incertitude de l'ordre de 8 à $11 \%$,

- les mesures n'ont été réalisées qu'avec une seule marque de gants atténuateurs. Il faudrait pouvoir les compléter avec tous les modèles qui sont actuellement sur le marché afin de vérifier si les résultats sont du même ordre,

- les mesures dans le rayonnement diffusé sont trop limitées. Il faudrait idéalement pouvoir réaliser d'autres séries,

- il pourrait également être utile de compléter ce travail en situation réelle d'exposition, en utilisant un gant sur lequel on aurait découpé la partie correspondant à la phalange distale de l'index, et en équipant l'index et le majeur de l'opérateur (main la plus exposée) de 2 dosimètres TLD stérilisés, positionnés sur les ongles.

\subsection{Impact des résultats}

En radiologie interventionnelle et au bloc opératoire, les gants radio-atténuateurs plombés sont utilisés pour diminuer l'exposition des mains des opérateurs aux rayons $\mathrm{X}$. 
Mais :

- Des mesures réalisées conjointement par le CHU de Clermont-Ferrand et l'IRSN, sur des installations de radiologie interventionnelle, montrent une augmentation de 13 à $42 \%$ de la dose reçue par les mains, dans le champ direct d'exposition.

- Le port de ces gants, dans le faisceau direct, entraîne également une augmentation de la dose délivrée au patient.

- Les gants radio-atténuateurs ne semblent être efficaces que dans le rayonnement diffusé ; cette atténuation n'a été observée que pour une tension donnée de l'ordre de $77 \mathrm{kV}$.

- L'usage de gants radio-atténuateurs plombés entraîne un faux sentiment de sécurité : les opérateurs se croient efficacement protégés et n'hésitent pas à mettre les mains sous le faisceau direct de rayons $\mathrm{X}$, majorant encore la dose qu'ils reçoivent.

- Les gants radio-atténuateurs induisent une perte de sensibilité tactile des sondes manipulées, et peuvent être à l'origine de dysfonctionnements durant l'examen, risquant ainsi d'augmenter la durée d'utilisation des rayons X.

Aussi, en regard de ces constats, l'atténuation de la dose reçue aux extrémités équipées de gants radio-atténuateurs, limitée au rayonnement diffusé, ne semble pas justifier pas leur utilisation et la dépense qu'elle induit.

\section{Conclusion}

Les mesures que nous avons réalisées font apparaître que l'utilisation des gants radio-atténuateurs plombés induit, dans le champ primaire d'exposition, une augmentation de la dose mesurée par les dosimètres TLD, qui oscille entre 14 et $42 \%$ en scopie, et entre 13 et $24 \%$ en graphie. Dans le rayonnement diffusé, les résultats montrent, en scopie, pour une tension donnée $(77-78 \mathrm{kV})$, une diminution d'un facteur 3 de la dose reçue par les dosimètres placés dans un gant. Mais cette expérimentation n'est pas le reflet de l'ensemble des pratiques et des régulations de tension auxquelles sont confrontés les praticiens en routine. Il est vraisemblable qu'avec une tension plus élevée, de l'ordre de 90 à 110 kV, comme cela est souvent le cas, la diminution de la dose avec les gants serait moins significative.

$\mathrm{Au}$ cours de procédures de radiologie interventionnelle, les opérateurs qui devront positionner leurs mains dans le faisceau direct de rayons $\mathrm{X}$ (ex. fistulographie), utilisent des gants radio-atténuateurs plombés, et pensent ainsi être efficacement protégés ; même si la procédure qu'ils réalisent ne nécessite pas le « passage » des mains dans le faisceau, ils n'hésiteront pas à le faire car « les gants les protègent ». Il semble donc qu'ils reçoivent à leur insu une dose majorée de rayons $\mathrm{X}$, liée à l'augmentation des paramètres de production d'image, due à la 
présence de plomb dans les gants radio atténuateurs (mode automatique de réglage des paramètres).

Pour que les gants soient efficaces dans le champ primaire d'exposition, il faudrait que les procédures soient réalisées en mode manuel, ce qui n'est pas envisageable.

La bonne pratique professionnelle, pour limiter efficacement la dose de rayons $\mathrm{X}$ reçue au niveau des mains, est, autant que faire se peut, de ne pas les positionner dans le faisceau direct de rayons $\mathrm{X}$.

\section{REFERENCE}

Wagner L.K., Mulhern O.R. (1996) Radiation-attenuating Surgical Gloves: Effects of Scatter and Secondary Electron Production, Radiology, 200, 45-48. 